\title{
A Synthetic, Closed-Looped Gene Circuit for the Autonomous Regulation of RUNX2 Activity during Chondrogenesis
}

\author{
Biming Wu ${ }^{1}$, Gurcharan $\operatorname{Kaur}^{1}$, Sunjana Murali², Thomas Lanigan³ ${ }^{3}$ Rhima M. Coleman ${ }^{1,4, *}$ \\ ${ }^{1}$ Department of Biomedical Engineering, University of Michigan, Ann Arbor, MI, USA \\ ${ }^{2}$ Program in Cellular and Molecular Biology, University of Michigan, Ann Arbor, MI, USA \\ ${ }^{3}$ Biomedical Research Vector Core, University of Michigan, Ann Arbor, MI, USA \\ ${ }^{4}$ Department of Mechanical Engineering, University of Michigan, Ann Arbor, MI, USA
}

\begin{abstract}
The transcription factor RUNX2 is a key regulator of chondrocyte phenotype during development, making it an ideal target for prevention of undesirable chondrocyte maturation in cartilage tissue engineering strategies. Here, we engineered an autoregulatory gene circuit (cisCXp-shRunx2) that negatively controls RUNX2 activity in chondrogenic cells via RNA interference initiated by a tunable synthetic CollOal-like promoter (cisCXp). The cisCXp-shRunx2 gene circuit is designed based on the observation that induced RUNX2 silencing after early chondrogenesis enhances the accumulation of cartilaginous matrix in 2D ATDC5 model. We show that the cisCXp-shRunx2 initiates RNAi of RUNX2 in maturing chondrocytes in response to the increasing intracellular RUNX2 activity without interfering with early chondrogenesis in ATDC5 cells. The induced loss of RUNX2 activity in turn negatively regulates the gene circuit itself. Furthermore, the efficacy of RUNX2 suppression from cisCXp-shRunx2 can be controlled by modifying the sensitivity of cisCXp promoter. Long-term $3 \mathrm{D}$ cultures of reprogrammed ATDC5 cells had increased matrix accumulation compared to naïve cells. Overall, our results demonstrate that the negative modulation of Runx2 activity with our autoregulatory gene circuit can reduce the effects of RUNX2 activity and enhance matrix synthesis in chondroprogenitor cells.
\end{abstract}

Keywords: cartilage; human mesenchymal stem cells; RUNX2; synthetic gene circuit

* Corresponding Author

1101 Beal Ave.

Ann Arbor, MI 48109

Email: rhimacol@umich.edu

Phone: +1-734-764-7740

Fax: +1-734-936-1905 


\section{INTRODUCTION}

Runt related transcription factor 2 (RUNX2) has a significant role in musculoskeletal development, including regulation of chondrocyte and osteoblast phenotype during endochondral ossification ${ }^{1,2}$. Multiple intracellular pathways, including those mediated by SMADs ${ }^{3}, \beta$-catenin ${ }^{4}$, and $\mathrm{p} 38^{5}$ signaling, are involved in chondrocyte maturation down the endochondral ossification pathway to hypertrophy. These pathways are regulated by various extracellular signaling molecules, including bone morphogenetic protein (BMP) ${ }^{6}$, transforming growth factor beta $(\mathrm{TGF} \beta)^{7}$, parathyroid hormone-related peptide $(\mathrm{PTHrP})^{8}$, Indian hedgehog $(\mathrm{IHH})^{9}$, fibroblast growth factor (FGF), and insulin like growth factor (IGF) ${ }^{10}$ as well as extracellular matrix (ECM) ligands such as hyaluronic $\operatorname{acid}^{11}$.

Synthetic regulation of the RUNX2 pathway has been a targeted for bone and cartilage tissue engineering, but its multifaceted functions in cell biology have led to mixed results. For example, blocking SMAD1/5/8 after chondrogenic differentiation of MSCs prevents terminal differentiation and mineralization while sustaining production of the articular cartilage structural macromolecules aggrecan and collagen type $\mathrm{II}^{3}$. Similarly, blocking the Wnt/ $\beta$-catenin pathway during chondrogenic culture of MSCs suppresses hypertrophy and in vivo mineralization ${ }^{16}$. Combined strategies using dynamic loading or hypoxic culture of MSCs in hydrogels containing ECM ligands can also regulate hypertrophic pathways ${ }^{15,17}$. However, it has also been shown that complete inhibition or silencing of these and other RUNX2-related pathways will prevent or significantly delay chondrogenesis ${ }^{3,16}$. While these studies have investigated the role of various pathways that regulate RUNX2 gene expression and activity in MSCs, the direct effect of stage-specific suppression of RUNX2 activity on cartilage tissue engineering outcomes has yet to be explored.

Gene silencing through RNA interference (RNAi) allows straightforward loss-of-function studies in mammalian cells to help understand the molecular mechanisms that underpin cartilage development. A virally introduced inducible short hair-pin RNA (shRNA) system provides temporal and reversible control of protein expression so that its activity may be evaluated in vitro over long culture periods, such as those required for tissue engineering. These insights could help us develop targeted silencing protocols in cartilage tissue engineering applications, which has advantages over broad spectrum inhibition of intracellular pathways that drive or respond to RUNX2 activity. However, chondrogenesis and subsequent maturation of MSCs is not a homogenous process. Therefore, inducible RNAi systems cannot be used to address the stage-specific effects of RUNX2 inhibition in cell populations that are at various stages of maturation. Furthermore, RNAi systems cannot be used to study the direct effect of exogenous cues on RUNX2-driven pathways.

Advances in synthetic biology allow us to reprogram cells with new functions by site-specific editing of the genome or combining basic genetic modules with well-characterized functions in novel ways to create gene circuits. The reprogrammed cells are then capable of autonomously detecting and adapting to changes in their environment. The utility of these techniques has been demonstrated in the production of stem cells with autoregulatory resistance to inflammatory stimulus ${ }^{18,19}$. Autoregulatory gene circuits can be used to evaluate the impact of exogenous cues on RUNX2 activity levels as well as regulate the maturation of chondrogenic cells mediated by RUNX2.

In this study, we sought to engineer an autoregulatory, closed-loop gene circuit to negatively regulate Runx2 activity in chondrogenic cells. Using an inducible RNAi system ${ }^{20}$, the effect of timing of Runx2 interference on the retention of articular cartilage-specific structural macromolecules was first assessed. Based on the outcomes of these experiments, we then designed an RNA-based regulatory unit containing a partial CollOallike promoter region with tunable activity that drives expression of short hairpin RNA (shRNA) for Runx2. The native CollOal promoter with the Runx 2 binding cis-enhancer region is $4.6 \mathrm{~kb}^{21}$ and are therefore difficult to fit into the limited encoding capacity of many viral vectors. Therefore, we constructed compact synthetic promoters de novo that contain multiple copies of Runx 2 cis-enhancers upstream of the essential regulatory elements to 
initiate transcription. This design permits tuning of the gene circuit's sensitivity to intracellular RUNX2 concentration as well as control of Runx2 activity levels. We characterized our circuits with in vitro specificity and sensitivity assays using the ATDC5 cell line. We then tested the hypothesis that the level of RUNX2 activity can be tuned by varying the number of $c$ is-enhancers incorporated into the promoter and that RUNX2 suppression level could increase the production and retention of structural macromolecules. Our results demonstrate that targeted disruption of Runx 2 activity to a specific chondrocyte maturation state can increase matrix production with limited off-target effects.

\section{METHODS}

\section{Experimental Design}

\section{Part 1: Design and Assessment of the Inducible shRunx2 System}

The experiments to characterize the effect of RUNX2 suppression on chondrogenesis and accumulation of cartilage-specific structural macromolecules are outlined in Fig. 1. ATDC5 cells were first transduced with lentiviruses expressing a TetOn inducible Runx 2 shRNA. Stable polyclonal cell populations were then established via antibiotic selection. RUNX2 suppression was then induced among these cells at day 0, 4, 7, 14, and 21 during a four-week chondrogenic program in monolayer culture followed by gene and protein expression profiling.

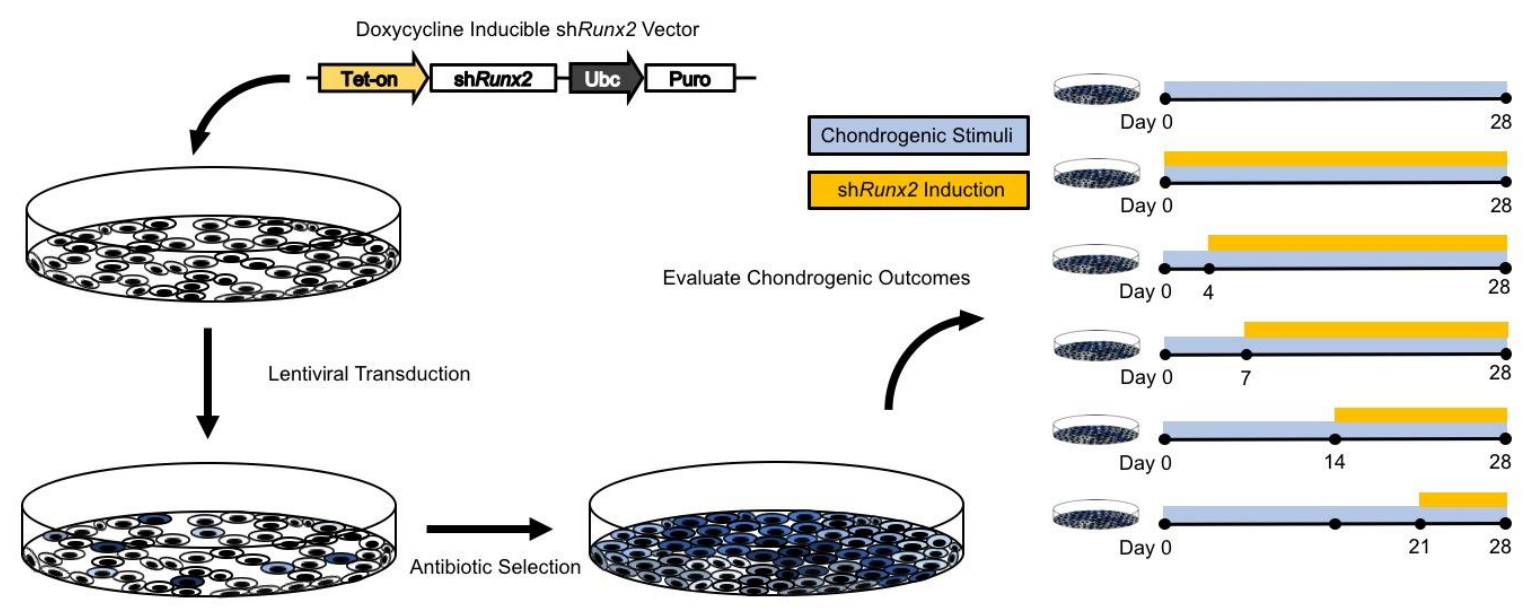

Figure 1. Experimental design of the inducible shRunx2 study.

\section{Part 2: Design and Assessment of the Autoregulatory shRunx2 Gene Circuit}

To enable autoregulatory RUNX2 suppression and characterize its effect on chondrogenesis, we engineered a gene circuit that induces the expression of Runx 2 shRNA in response to increasing intracellular concentrations of active RUNX2 using a synthetic CollOal-like promoter. Chondrogenesis of a polyclonal ATDC5 cell population stably expressing such a gene circuit was evaluated in both 3-week monolayer culture and 5-week pellet culture (Fig. 2). 


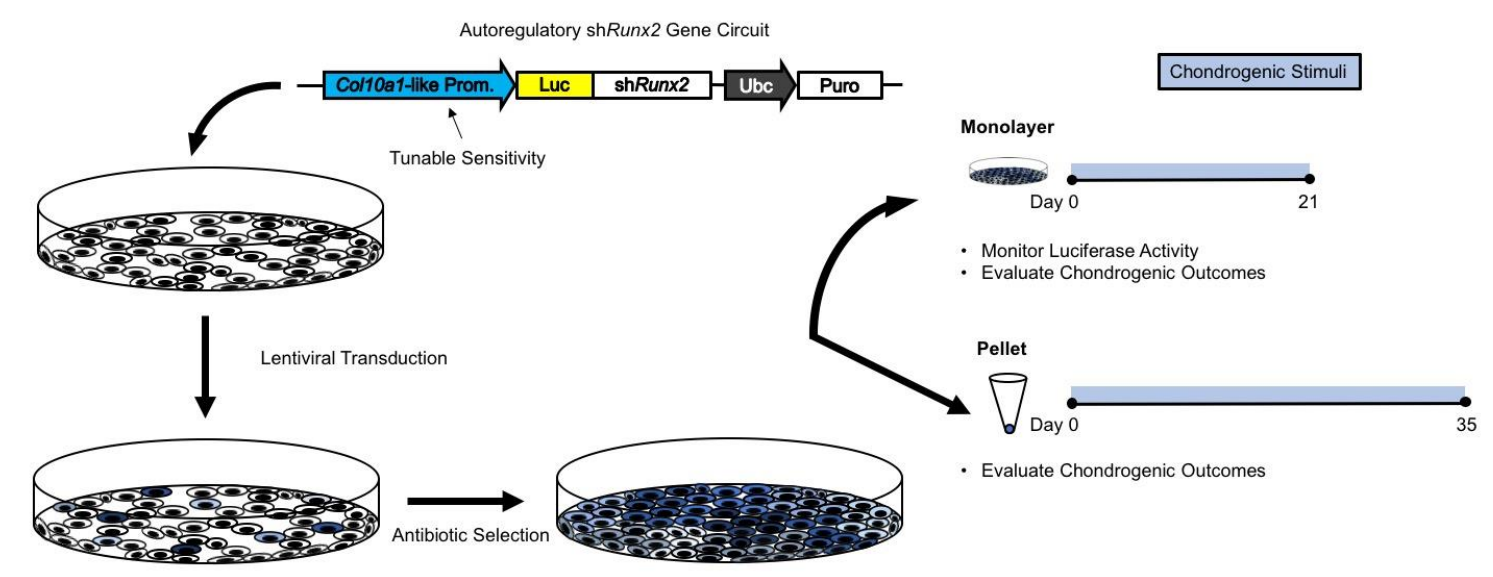

Figure 2. Experimental design of the autoregulatory shRunx2 gene circuit study.

\section{Chondrogenic Cell Cultures}

ATDC5 (Sigma; RRID:CVCL_3894) cells were used to model the transition of different phases of endochondral ossification as this cell line can recapitulate chondrogenic maturation in both monolayer (2D) and pellet (3D) cultures on an accelerated timescale ${ }^{22-24}$. After expansion, cells were transduced with the vectors described below. Chondrogenic behavior of these cells and their mineralization response to exogenous phosphate were carefully characterized in a previous study, which also described the detailed chondrogenic culture protocol used in this study $^{25}$. Briefly, chondrogenesis in 2D cultures of transduced or wild-type cells was initiated by addition of $1 \%$ ITS+ Premix (Corning) and $50 \mu \mathrm{g} / \mathrm{ml}$ L-ascorbic acid-2-phosphate upon 100\% confluence. In 3D cultures, cell pellets containing $2.5 \times 10^{5}$ cells were cultured in round-bottomed polypropylene 96-well-plates and media was changed every 2 days for 35 days.

\section{Lentiviral Vector Design and Synthesis}

\section{Doxycycline Inducible shRunx2 Vector}

The inducible system that drives RUNX2 knockdown was modified from the pINDUCER lentiviral toolkit originally developed by Meerbrey et $a l .{ }^{20}$. pINDUCER13 was acquired from Addgene (plasmid \# 46936) for further modification as such a lentiviral vector conveniently contains a Tet-on inducible cassette that co-expresses luciferase and shRNA sequence as well as a cDNA sequence of the puromycin resistance gene that was constitutively active (Fig 3). Three shRNA sequences targeting murine/human Runx2 from RNAi Codex ${ }^{28}$ were screened for RUNX2 knockdown via western blotting and shRunx2 sequence \#294717 (5'-TGCTGTTGACAGTGAGCGCCGAATGGCAGCACGCTATTAATAGTGAAGCCACAGATGTATTAATAGCGT-

GCTGCCATTCGATGCCTACTGCCTCGGA-3') was further selected for downstream vector synthesis.

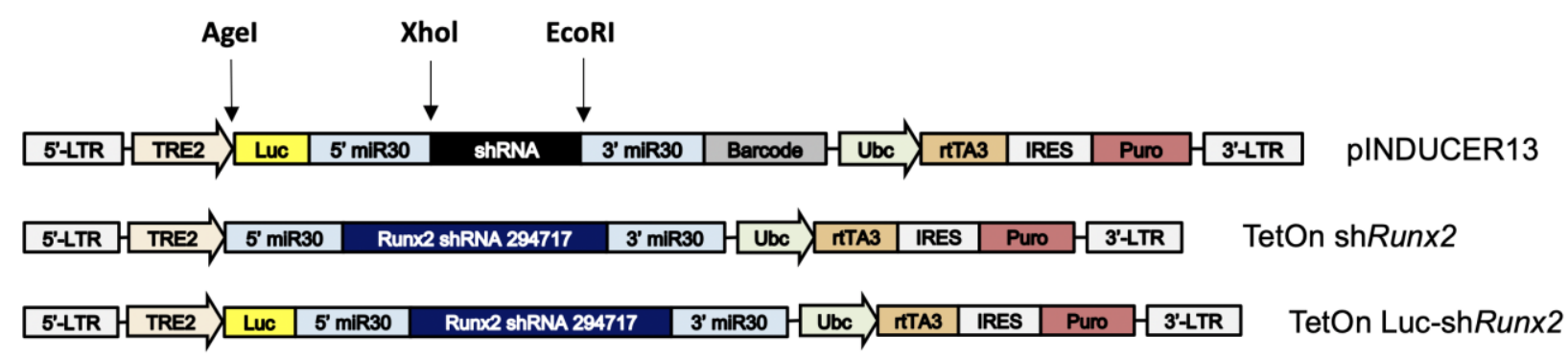

Figure 3. Diagrams of pINDUCER13 and TetOn inducible shRunx2 vectors. TRE2, tetracycline response element 2 promoter; Luc, luciferase; Ubc, Ubiquitin C promoter; rtTA3, reverse tetracycline-controlled transactivator 3; Puro, puromycin resistance. Diagram of pINDUCER13 is adapted from Meerbrey et $a l^{20}$. 
Two inducible shRunx2 vectors (TetOn-Luc-shRunx2 \& TetOn-shRunx2) were created. TetOn-Luc-shRunx2 was first created by sub-cloning PCR-amplified shRunx2 \#294717 into TetOn-inducible cassette of pINDUCER13 via XhoI/EcoRI sites. To remove the luciferase cDNA from TetOn-Luc-shRunx2, miR30-shRunx2-miR30 sequence was PCR amplified with the addition of an AgeI site upstream of the 5' end. The PCR product was subsequently sub-cloned into the TetOn-Luc-shRunx2 vector to replace the existing Luc-miR30-shRunx2-miR30. In addition, two scramble vectors (TetOn-Luc-scramble \& TetOn-scramble) were synthesized to serve as experimental controls (Table S1). Expression of short hairpin sequences was induced by the addition of $(0.5 \mu \mathrm{g} / \mathrm{ml})$ of doxycycline (Dox) beginning on the day indicated and continued to the end of culture.

\section{Autoregulatory cisCXp-shRunx2 gene circuit}

The autoregulatory cisCXp-shRunx2 gene circuits rely on a CollOal-like promoter to drive RUNX2 knockdown via expression of shRunx2 sequence. Colloal-like promoters with a different number of cis enhancers were first reported by Zheng et al. ${ }^{21}$; single copies of 1 cisCXp promoter which consists of one cis enhancer (-4296 to -4147 bp) upstream of the Colloal basal promoter (-220 to $110 \mathrm{bp}$ ) were acquired from IDT technology (Fig. 4). To create 1cisCXp-shRunx2, the 1cisCXp promoter was PCR amplified using corresponding primers (Table S2) so that both 5'- and 3'-ends of the reaction products have an overlap of > 30 nucleotides with the backbone of TetOnLuc-shRunx2 vector after TRE2 promoter was removed by restriction digestion with NheI/AgeI. The linearized Luc-shRunx 2 vector and 1cisCXp promoter were further assembled together using Gibson Assembly Kit (NEB).

To create 2cisCXp-shRunx2, single copies of 150-bp cis enhancer were acquired from IDT technology. PCR amplifications of both the cis-enhancer and 1 cis CXp promoter were performed using corresponding primers (Table S3) so that overlaps of $>30$ nucleotides are created between the reaction products to support the sequential assembly of linearized Luc-shRunx2 vector, cis enhancer, and 1cisCXp. Similarly, 3cisCXp-shRunx2 was assembled from the three components described above as well as an additional PCR product that inserts an extra copy of cis enhancer in between the first cis enhancer and 1cisCXp promoter. Correct cloning was confirmed by Sanger sequencing at University of Michigan Sequencing core.

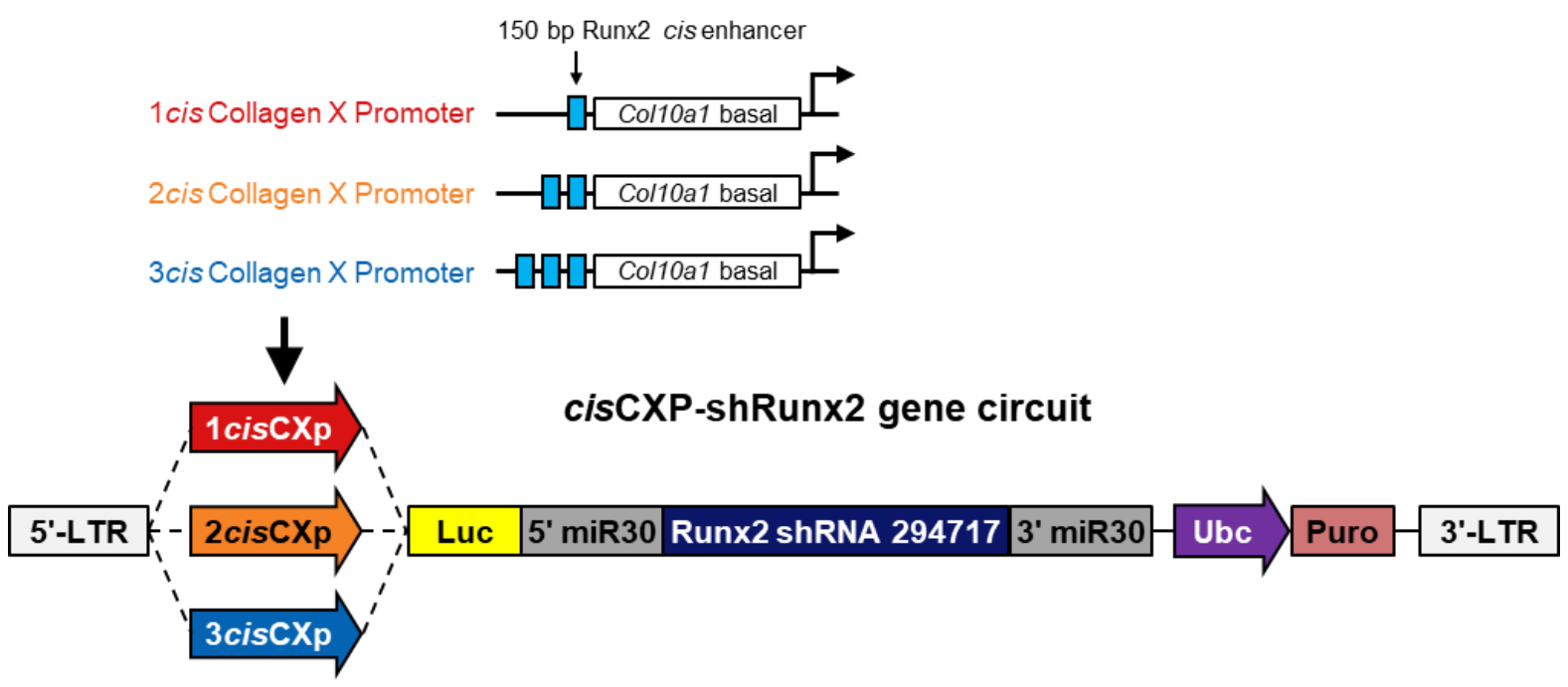

Figure 4. Diagrams of CXp promoter cisCXp-shRunx2 gene circuits. 1-, 2-, and 3cisCXp promoters contains one, two, and three copies of 150bp CollOal cis-enhancer upstream of CollOal basal promoter.

\section{Lentiviral Transduction and Stable Selection}

To establish ATDC5 cell lines that express the vectors of interest, lentiviral supernatant of chosen plasmid vectors was produced by the University of Michigan Vector Core. Twenty-four hours prior to transduction, cells were 
plated in individual wells of a 6-well plate at a density of 2000 cell $/ \mathrm{cm}^{2}$. Proliferating cells were transduced at multiplicity of infection $(\mathrm{MOI})=1$ with lentiviral supernatant of TetOn-shRunx2, cisCXp-Luc-shRunx2 gene circuits, and corresponding scramble vectors in the absence of serum for 48 hours. Polyclonal populations of cells stably expressing the chosen vectors were subsequently selected with continuous treatment of puromycin $(2 \mu \mathrm{g} / \mathrm{ml})$ for 10-14 days for ATDC5 cells. Stably transduced ATDC5 cells were subcultured for one additional passage prior to each experiment.

\section{Luciferase Assay}

Luciferase activity of 2D and 3D cultures in multi-well plates was measured once daily beginning on day -1 or 0 . Thirty minutes prior to each measurement, concentrated D-luciferin stock $(1 \mathrm{mg} / \mathrm{ml})$ was added to existing culture media to achieve a final concentration of $150 \mu \mathrm{g} / \mathrm{mL}$ and gently mixed. Cultures containing luciferin were then incubated at $37^{\circ} \mathrm{C}$ for 30 minutes before measured using SYNERGY H1 microplate reader (BioTek). Each sample was measured three times ( 1 second/read) and the means were normalized to the day -1 values and expressed as relative luminescence units (RLU). Media was replaced in measured cultures immediately after reading.

\section{Biochemical Analysis}

To assess the accumulation of cartilage-specific matrix in both 2D and 3D cultures, the level of sGAG of each sample was quantified using DMMB assay as previously described ${ }^{25,29}$. Specifically, differentiated cell masses from 2D cultures or pellets from 3D cultures were washed with ice-cold PBS before being digested with $1 \mathrm{mg} / \mathrm{ml}$ proteinase $\mathrm{K}$ in $200-500 \mu \mathrm{l}$ ammonium acetate $(0.1 \mathrm{M})$ at $50^{\circ} \mathrm{C}$ for 16 hours. sGAG content in the digested lysate of each sample was subsequently determined by comparing its DMMB reading to a standard curve. The DNA content of each sample was measured using Hoechst 33258 dye (Sigma) to normalize its corresponding sGAG content ${ }^{30}$.

\section{Gene Expression Analysis}

Analysis of gene expression was performed as previously described ${ }^{4}$. Total RNA of both 2D and 3D cultures were extracted using TRI Reagent ${ }^{\circ}$ RT (Molecular Research Center). Cell masses from each individual well of 12-well plate were collected as one 2D sample. Four pellets were combined to generate $13 \mathrm{D}$ sample. Extracted RNA were reverse-transcribed into cDNA using High-Capacity cDNA Reverse Transcription Kit (Life Technologies). Relative expression levels were calculated as $x=2^{-\Delta \Delta C_{T}}$, in which $\Delta \Delta C_{T}=\Delta C-\Delta E, \Delta E=$ $C_{T}^{e x p}-\operatorname{avg} C_{T}^{h k}$ at the time-point of interest, $\Delta C=C_{T}^{e x p}-\operatorname{avg} C_{T}^{h k}$ at day 0 , and $\operatorname{avg} C_{T}^{h k}$ is the average $C_{T}$ value of two housekeeping genes (Hprt and Ppia). The forward and reverse primer sequences for all genes are listed in Table S2.

\section{Histological Analysis}

Monolayer cultures were washed twice with PBS and fixed in 70\% ethanol at room temperature for one hour. Fixed pellets were washed with $70 \%$ ethanol, embedded in paraffin wax, and then $7 \mu \mathrm{m}$ sections were taken. Sections were stained with Alcian blue (1\% in 3\% Acetic Acid, Poly Scientific) at $4{ }^{\circ} \mathrm{C}$ for 16 hours and then counter-stained with Fast Nuclear Red. Stained sections were imaged using bright field microscopy.

\section{Immunohistochemistry}

The pellet sections were deparaffinized in xylene and rehydrated in successive dilutions of ethanol. Slides were then blocked in $3 \% \mathrm{H}_{2} \mathrm{O}_{2}$ for 10 minutes. This was followed by Antigen retrieval with heated Retrievagen A (BD 
Biosciences, Cat\# 550524) for 20 minutes. Once cooled to room temperature, the samples were then permeabilized using 0.5\% TBS-TX100, blocked with 10\% Goat serum, 1\% BSA in 0.1\% TBST for 2 hours at room temperature, and then incubated overnight in RUNX2 primary antibody solution (ABclonal Cat\# A2851; 1:100 dilution in $10 \%$ Goat serum in $0.1 \%$ TBS-TX100) at $4^{\circ} \mathrm{C}$. After washing, samples were incubated with Goat Anti-Rabbit IgG H\&L (HRP) preabsorbed secondary antibody (Abcam, Cat\# ab97080; 1:500 dilution in $10 \%$ Goat serum in $0.1 \%$ TBS-TX100) followed by incubation with 3,3'Diaminobenzidine DAB substrate (Abcam Cat\# ab64238) for 10 min at the room temperature. Samples were then counterstained with Hemotoxylin and cover-slipped mounting media.

\section{Western Blot Analysis}

Whole cell extracts of 2D cultures from one well of a 6-well plate were prepared using RIPA Lysis and Extraction Buffer (Thermo Scientific) supplemented with protease inhibitor cocktail (Sigma) using the Micro Tube Homogenizer (Thermo Scientific). Homogenized lysate was rotated at $4{ }^{\circ} \mathrm{C}$ for one hour and centrifuged at $12,000 \mathrm{~g}$ for 10 minutes to remove cellular debris. Total protein content within each sample was determined with the Pierce BCA Protein Assay Kit (Pierce). Proteins (5-15 $\mu \mathrm{g}$ ) were separated on a 10\% NuPAGE Bis-Tris Protein Gel and then transferred to a polyvinylidene difluoride membrane (Millipore). Membranes were blocked with $5 \%$ BSA made up in Tris-buffered saline-Tween 20 (TBS-T, 0.1\% Tween 20) for 1 hour at room temperature. Following blocking, membranes were incubated in TBS-T, $0.1 \%$ Tween 20 and $5 \%$ BSA overnight at $4{ }^{\circ} \mathrm{C}$ with anti-Runx2 antibody (RUNX2 D1H7 Rabbit mAb, Cell Signaling Technology, 84866 1:2000) and anti- $\beta$-actin antibody (Rabbit Anti-beta Actin, Abcam, ab119716, 1:5000). Secondary incubation was performed at room temperature for 1 hour using WesternSure® Goat anti-Rabbit HRP (LiCor, 926-80011 1:20000). Positive staining was visualized using the LiCor WesternSure® PREMIUM Chemiluminescent Substrate and quantified using the LiCor Image Studio.

\section{Statistics}

Statistical analyses were performed in GraphPad Prism version 7 for Windows (GraphPad Software, La Jolla California USA) using one or two-way analysis of variance followed by Tukey's post-hoc test. P-values less than 0.05 were considered statistically significant. All errors bars are the mean \pm the standard error of the mean.

\section{RESULTS}

\section{Part 1: Design and Assessment of the Inducible shRunx2 System}

\section{Constitutively Silencing of RUNX2 Inhibits Chondrogenesis}

To examine the effect of constitutive RUNX2 silencing on in vitro differentiation of chondroprogenitors, we induced chondrogenesis in monolayer cultures of ATDC5 cells stably expressing TetOn-shRunx2 (shRunx2 cells) in presence of doxycycline (Dox) and evaluated matrix accumulation and expression levels of chondrogenic markers after 14 days. In cultures treated with Dox, RUNX2 protein was depleted in shRunx2 cells by day 7 and remained downregulated through day 14 (Figure 5a). With Dox treatment, there was also no detectable amounts of matrix accumulation in shRunx 2 cells by day 14, while the scramble controls had successfully formed Alcian blue-positive nodules (Figures $5 \mathrm{~b}$ and c). The expression of mRNA for Acan and Col2al was also significantly inhibited with Dox treatment of shRunx2 cells (Figure 5d), suggesting that constitutive RUNX2 suppression interfered with early chondrogenesis. 
a



C

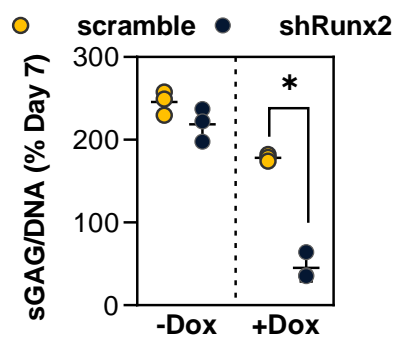

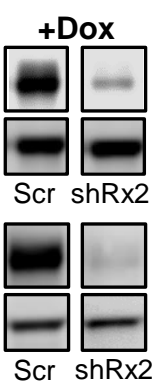

d

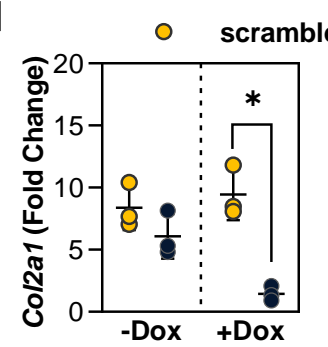

b

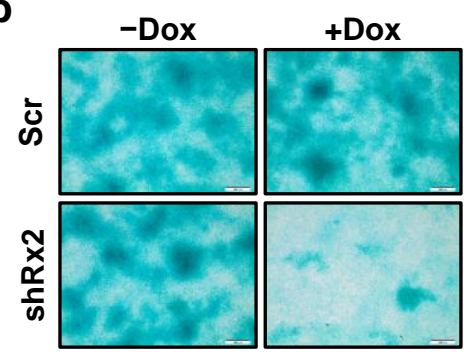

Figure 5. Effects of constitutively active RUNX2 silencing on early chondrogenesis. ATDC5 cells expressing Tet-on shRunx2/scramble were differentiated in the presence $(+)$ or absence $(-)$ of 0.5 $\mu \mathrm{g} / \mathrm{ml}$ doxycycline for 14 days. (a) Western blot analysis of RUNX2 and $\beta$-actin at day 7 and 14 . Alcian blue staining (b) and \% sGAG accumulation relative to samples at 7 days without Dox (c) at day 14. (d) Fold change in mRNA expression for aggrecan and collagen type II at day 14. Polyclonal populations were established by combining selected cells from two independent transduction experiments (two viral batches, $n=3$ ). All data represented as mean \pm SD. Significant difference is indicated by $* \mathrm{p}<0.05$.

\section{Stage-dependent Effect of RUNX2 Silencing on Chondrogenesis}

RUNX2 suppression in chondroprogenitors as they mature through the stages of endochondral ossification was examined by adding Dox continuously after the following time-points of culture that correlate with specific stages of chondrocyte maturation, as determined in preliminary studies (Fig. 6a and Fig. S1): minimum RUNX2 protein expression (D4), just prior to rapid increase in RUNX2 protein expression (D7), peak gene expression of Colloal $(\mathrm{D} 14)^{25}$, and the peak of RUNX2 protein expression (D21). These were compared to cultures that received no Dox or were treated with Dox from day 0 (D0). Dox induction in monolayer suppressed RUNX2 protein levels by $20-60 \%$ in all cultures by day 28 (Fig. 6b). Matrix accumulation was also increased by 1.9 -fold, 2.14 -fold and 1.75 -fold on day 28 relative to scramble controls and the no Dox group, when RUNX2 suppression was induced from Day 4, 7, and 14, respectively (Fig. 6c). There was no difference between the scramble and no Dox groups.

a

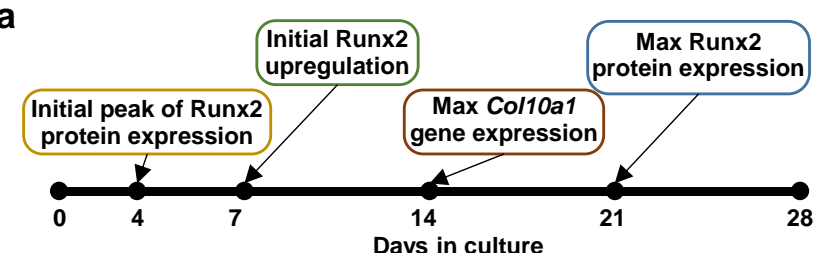

b



C



d

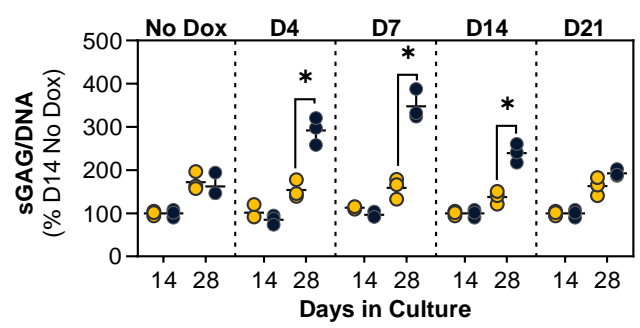

Figure 6. Effects of RUNX2 silencing at different maturation stages of chondrogenesis. (a) The time-course of chondrocyte maturation and relative Runx2 protein expression in monolayer cultures of ATDC5 cells. (b) Runx2 protein band intensity normalized by $\beta$-actin. The plot shows the percent increase in protein expression compare to just prior to induction of chondrogenesis (day -1). (c) Relative RUNX2 protein expression in TetOn-shRunx2 cells in response to Dox treatment from different time points of chondrogenic differentiation relative to the No Dox controls. (d) Fold change relative to D14 No Dox group in sGAG accumulation normalized to DNA content, after 14 and 28 days in culture. Data represented as mean \pm SD. Polyclonal populations were established by combining selected cells from two independent transduction experiments (two viral batches, $\mathrm{n}=3$ ). Significant difference between TetOn shRunx2 and scramble groups at each time point is indicated by $* * \mathrm{p}<0.005$ or $* * * \mathrm{p}<0.0001$. 
We next examined the effect of Runx2 suppression beginning on D4 or D7 on genes associated with chondrocyte hypertrophy, ECM production, and ECM turnover (Fig 7). Runx2 suppression reduced expression of the hypertrophy marker CollOal by 2-fold at 14 days of culture. This level was maintained through day 28. Expression of Mmp13, another significant marker of hypertrophy, was downregulated 5-20-fold by Runx2 suppression independent of when Dox treatment began. When RUNX2 was suppressed beginning on day 4 or day 7, gene expression of the chondrogenic ECM markers aggrecan and collagen II were not significantly reduced compared to controls that did not receive Dox. Runx2 suppression beginning at day 4 had no effect on Adamts5 gene expression while delaying suppression until day 7 lead to increased expression of this metalloproteinase. Suppression beginning at either time point had no effect on Adamts4 expression.
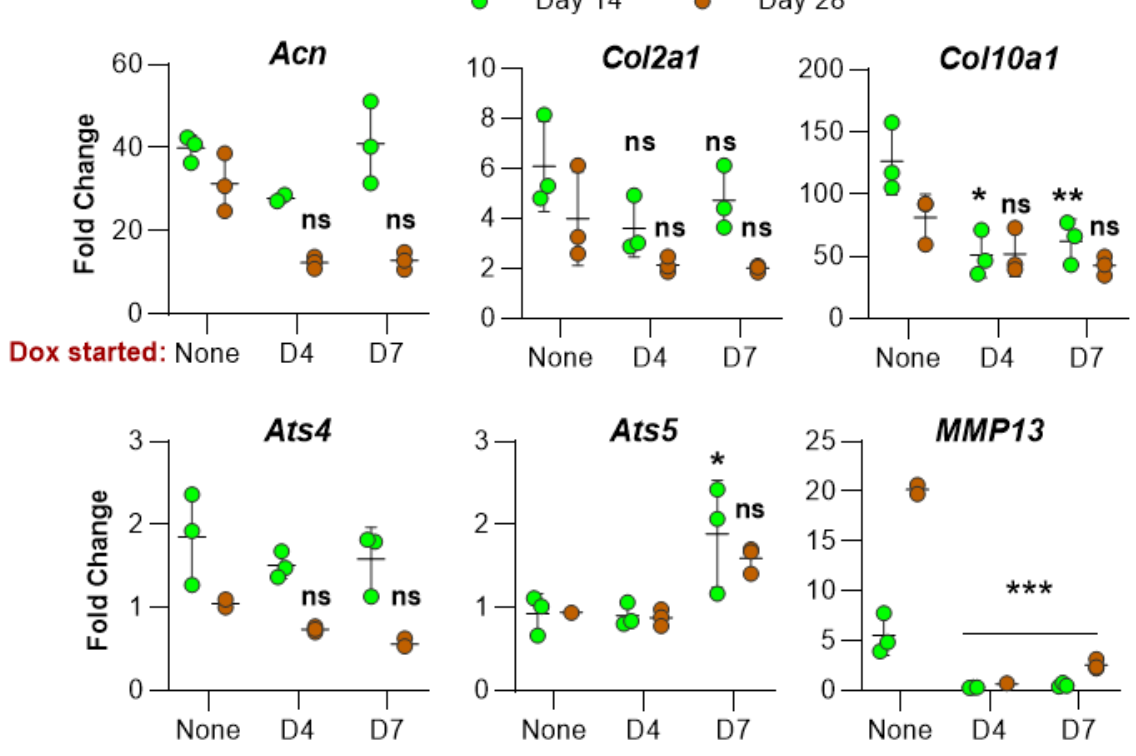

Figure 7. Effects of delayed RUNX2 silencing on gene expression of chondrogenic markers and matrix degrading enzymes. Quantification of mRNA expression of Acn, Col2al, Colloal, Adamts4, Adamts5, and Mmp13 at day 14 and 28 in cells with inducible expression of shRUNX2 beginning on D4 or D7. Polyclonal populations were established by combining selected cells from two independent transduction experiments (two viral batches, $n=3$ ). All data represented as mean \pm SD. Significant difference from samples that did not receive Dox (None) at the specified time-point is indicated by $* \mathrm{p}<0.05$, ** $\mathrm{p}<0.005$, or $* * *$ $\mathrm{p}<0.0001$.

This inducible study indicates that suppression of RUNX2 at specific points of chondrocyte maturation has a differential impact on matrix accrual of cartilage-specific structural macromolecules and regulation of metalloproteinases expression. Therefore, persistent and broad-spectrum silencing of RUNX2 could have deleterious effects on chondrogenesis and matrix production in heterogeneous populations of chondroprogenitors at various stages of maturation. This makes it difficult to use inducible silencing to optimally target its suppression. Next, we sought to engineer an autoregulatory genetic modification that would allow the cells to independently regulate their response to exogenous cues that raise intracellular RUNX2 levels.

\section{Part 2: Design and Assessment of the Autoregulatory shRunx2 Gene Circuit}

\section{Engineering of a Synthetic Coll0al-like Promoter}

To engineer a RUNX2-responsive promoter that is specific to chondrogenic cells, we assembled the 150bp RUNX2-binding cis-enhancer of the CollOal promoter upstream of a truncated basal sequence from the same promoter (ncisCXp; Fig. 4). To demonstrate that this promoter is specific for chondrogenic cells undergoing hypertrophic maturation, a promoter containing 1 cis-enhancer (1cis CXp) was used to drive the expression of luciferase or eGFP (Fig. 8a). Luciferase activity mirrored the time-course of Colloal gene expression (Fig. 8b) in chondrogenic ATDC5 cells: minimal until day 6 followed by a rapid increase to maximum level at day 10 and sustained thereafter (Fig. 8c). eGFP fluorescence was only seen in cartilaginous nodules starting on day 7 (Fig. 8d). Little eGFP fluorescence was observed in cells that were not chondrogenically induced nor ones that had not yet formed cartilaginous nodules. Western blot analysis at day 14 showed that eGFP protein was solely expressed 
in cells that were exposed to chondrogenic stimuli (Fig. 8e). Taken together, these data illustrate that the 1 cisCXp promoter has phenotype-specificity similar to the endogenous CollOal promoter, its transcriptional activity is limited to differentiated ATDC5 cells, and increases as these cells transition to the hypertrophic phenotype.

a

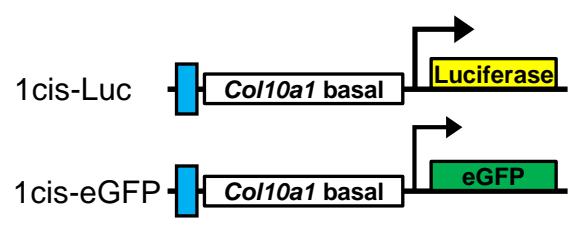

d
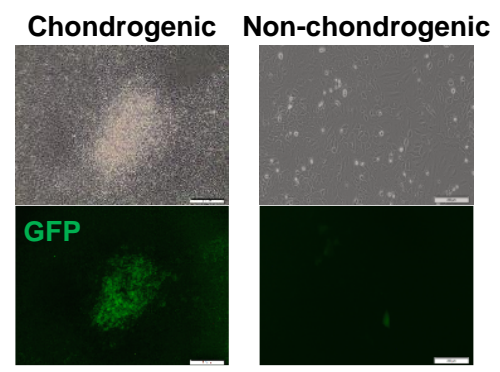

b

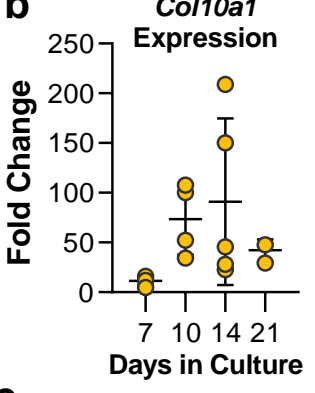

e
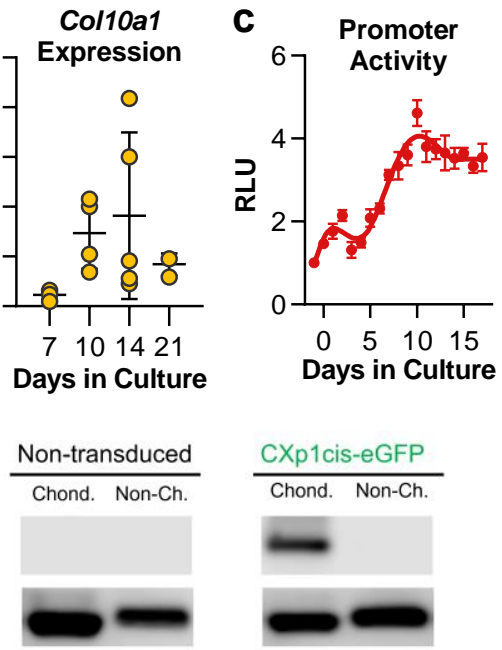

Figure 8. Specificity of the gene circuit. (a) Diagrams of 1cisCXp-Luc and 1cisCXp-eGFP vectors used to evaluate the activity and chondrogenic specificity, respectively, of the engineered Colloallike promoter. (b) Quantification of mRNA expression of CollOal in non-transduced ATDC5 monolayer cultures over 21 days of chondrogenic differentiation (two independent experiments, $\mathrm{n} \geq 5$ ). (c) Luciferase activity from ATDC5 cultures expressing 1cis-Luc over 21 days of differentiation relative to day -1 (three independent experiments, $n=6$ ). (d) Bright field and fluorescent imaging of ATDC5

cells transduced with 1 cis CXp-eGFP showing that GFP is localized to cartilage nodules of chondrogenic cultures at the onset of Col10al mRNA upregulation (day 7), demonstrating the specificity of the gene circuit activity to chondrogenic cultures. (e) Western blot for eGFP and $\beta$-actin (internal control) levels after 14-day chondrogenic cultures in non-transduced ATDC5 cells compared to cells transduced with 1cisCXp-eGFP, also demonstrating gene circuit specificity.

\section{Col10a1-like promoter can be engineered to tune the activity of RUNX2 during chondrogenesis}

To determine whether the CollOal-like promoter could be used to provide self-regulated silencing of RUNX2 and to test the hypothesis that the level of RUNX2 suppression could be tuned by varying the number of cisenhancers, we monitored the activity of cisCXp-shRunx2 gene circuits during the chondrogenesis of polyclonal ATDC5 cell populations stably expressing these vectors (Fig. 4). We similarly synthesized 2cis-shRunx2 and 3 cis-shRunx2 gene circuits and their corresponding scramble control vectors. During the 21-day monolayer chondrogenic differentiation assay, luciferase activity was used as a surrogate measurement of promoter activation.

Luminescence was minimal in cells expressing the 1cis-shRunx2 vector during the first 6 days, similar to that in cells transduced with 1 cis-scramble vector. From day $7, \operatorname{sh} R u n x 2$ containing cells exhibited a significantly lower level of luminescence than the scramble controls (Fig. 9a and b). While the total luminescence from both groups fluctuated throughout further differentiation, a stable relative decrease in RUNX2 activity of $18.1 \pm 5.1 \%$ compared to scrambled controls was reached under the 1 cis-shRunx2 vector. Similarly, gene circuits containing the 2 cis CXp promoter exhibited the same level of minimal activity as 1cisCXp during the first three days of chondrogenic differentiation. Thereafter, the 2 cisCXp promoter drove the total gene circuit activity in 2 cisshRunx2 cultures to equilibrate at a level that was $30.4 \pm 4.2 \%$ lower than the scramble controls. The addition of a third cis-enhancer exhibited a more prominent reduction $(77.5 \pm 2.6 \%)$ in gene circuit activity when compared to the scramble control starting at the onset of chondrogenesis. Gene expression of Colloal was significantly downregulated only in 3cis-shRunx2 expressing cultures at D14 (Fig. 9c). Significantly more sGAG was accumulated by 2 cis-shRunx 2 and 3cis-shRunx 2 cultures at day 14 (Fig. 9d). Alcian blue stained sections showed an increase in the staining area of cartilage nodules. RUNX2 staining decreased with increasing numbers of cisenhancers compared to the scramble control (Fig. 9e). 
a

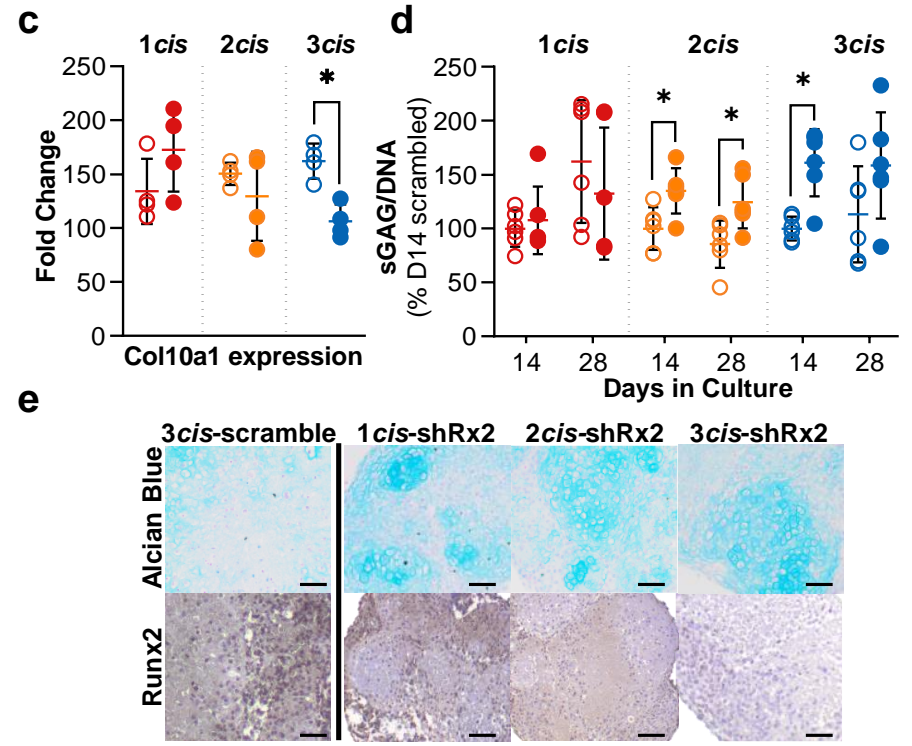

Figure 9. Activity of RUNX2 Suppressing Gene Circuits and Their Effect on Chondrogenesis. (a) Luminescence measured in 21day chondrogenic cultures expressing 1cisCXp-shRunx2/scramble, 2cisCXp-shRunx2/scramble, 3cisCXp-shRunx2/scramble as a measure of RUNX2 activity. (b) Relative activity of each gene circuit was calculated by normalizing to activity of corresponding scramble controls at each time point. (c) Quantification of mRNA expression (relative to day 0 and the housekeeping genes Hprt and Ppia) of the RUNX2 target gene, Colloal, at day 14. (d) Fold change in sGAG accumulation normalized to DNA content at day 14 and 28. (e) Staining for SGAG and Runx2 in ATDC5 pellet cultures after 28 days of culture. Data represented as mean \pm SD. Significant difference scramble (open circles) and shRunx 2 (closed circles) at each time point ( $\mathrm{n}=6$ from two independent transduction experiments) is indicated by $* \mathrm{p}<0.05$.

\section{DISCUSSION}

Here, we demonstrate that the loss of RUNX2 function during chondrogenesis of chondroprogenitor cells can elicit distinct cellular responses at different stages of differentiation. Using a 2D ATDC5 model with inducible shRunx2 expression, we show that RNAi of Runx2 in the undifferentiated cells inhibits differentiation into chondrocytes. However, induced expression of shRunx 2 after the pre-chondrogenic proliferation phase or early chondrogenesis enhances the accumulation of cartilaginous matrix. We further demonstrate that the synthetic Colloal-like promoters can initiate RNAi of Run 2 in chondrocytes that are transitioning to the pre-hypertrophic phenotype in response to the increasing intracellular RUNX2 activity without interfering with early chondrogenesis. The induced loss of RUNX2 function in turn negatively regulates the activity of the cis-shRunx2 gene circuit to resist upregulation of RUNX2 during hypertrophy and maturation-associated matrix degradation. Together, our findings highlighted three key features of the cis-shRunx2 gene circuit: 1) phenotype-specific activation of RNAi, 2) closed-loop intracellular negative-feedback regulation of RUNX2 activity, and 3) tunable levels of RUNX2 repression. Such features of the gene circuits can help chondroprogenitors accumulate cartilaginous matrix during chondrogenesis.

Unlike the well-established role of RUNX2 in driving chondrocyte maturation, its involvement in early events of chondrogenesis remains unclear. Progenitor cells within the prechondrogenic condensation first proliferate before committing to chondrogenic differentiation ${ }^{33-35}$. Runx 2 expression has been detected within these condensations ${ }^{36-38}$. Recently, Dexheimer et al. showed that this transient phase of proliferation is required for in vitro chondrogenesis of human MSCs after cells are condensed into a micromass pellet ${ }^{39}$. In our study, when chondrogenesis is induced in confluent monolayer ATDC5 cultures, differentiation initiates with a similar phase of proliferation that overrides contact inhibition during the first 4 days, which does not occur under the RNAi of Runx2 (Fig S1). In accordance with our observations, Akiyama et al. also showed that introduction of the dominant negative form of RUNX2 in ATDC5 cells inhibits cellular condensation and subsequent 
chondrogenic differentiation ${ }^{40}$. Similar suppression of chondrogenesis is also observed when Zfp521, an inhibitor of RUNX2, is overexpressed in ADTC5 cells ${ }^{41}$. However, it is worth noting that Runx $2^{-/}$mice do form cartilaginous skeleton ${ }^{42,43}$, suggesting that the in vivo there are redundant pathways that participate in cartilage formation and that in vitro chondrogenesis is not reproducing this process. Nonetheless, constitutively active RNAi of Runx2 is not suitable cartilage tissue engineering.

As chondrogenesis proceeds, the role of RUNX2 changes $^{44}$. Delayed RUNX2 silencing in chondroprogenitors after the pre-chondrogenic proliferation phase does not interfere with their further chondrogenic progression; instead it increases the amount of matrix accumulated by these differentiated chondrocytes and decreases the gene expression of MMP13. After pre-chondrogenic proliferation in vivo, Nkx3.2-mediated repression of Runx2 promotes early chondrogenesis by activating Sox $9^{45}$. These findings are analogous to our observation of the transient upregulation of RUNX2 prior to the elevated levels of Col $2 a 1$ and Acan expression. They also likely explain why loss of RUNX2 function after day 4 no longer blocks chondrogenesis in ATDC5 cells. As in growth plate chondrocytes ${ }^{46}$, the low protein expression of RUNX2 is not permanent in the ATDC5 model. Quickly following the upregulation of Col2al and Acan, protein expression of RUNX2 rose and drove differentiated chondrocytes to the pre-hypertrophic and then hypertrophic phenotypes. As expected, induced RUNX2 silencing at this stage of chondrocyte maturation decreased mRNA levels of both CollOal and Mmp13. MMP13 can degrade both aggrecan ${ }^{47}$ and type II collagen ${ }^{48}$ and downregulation of $M m p 13$ due to the loss of RUNX2 function has been reported to reduce the breakdown of these structural molecules in Runx 2 deficient mice ${ }^{49}$. This is consistent with our observation that RUNX2 silencing leads to an increased level of matrix accumulation after control groups undergo hypertrophy.

The cisCXp-shRunx2 gene circuit relies on its synthetic CollOal-like promoter to induce hypertrophyspecific RUNX2 silencing. The specific expression of CollOal in pre-hypertrophic and hypertrophic chondrocytes requires the binding of RUNX2 in addition to the recruitment of the general transcription factors near the transcription start site $21,52,53$, which usually reside within the basal region of mammalian polymerase II promoters. The 330-bp Colloal basal promoter we incorporated contains a highly conserved sequence that precisely describes the transcription start site of Colloal across species ${ }^{21}$, providing the DNA template that supports the assembly of the RNA polymerase II transcription initiation complex. Meanwhile, the two putative tandem-repeat within the 150-bp cis-enhancer ensures direct binding of RUNX2 ${ }^{21,54}$. As a result of the cooperative actions of these two regulatory elements, all three versions of cis promoter are sufficient to direct hypertrophyspecific transcription resembling the endogenous CollOal promoter in differentiating chondrocytes. Therefore, the suppression of early chondrogenesis can be avoided as the loss of RUNX2 function does not occur until progenitors fully differentiate into chondrocytes and transition to pre-hypertrophy.

The negative feedback motif of cisCXp-shRunx2 utilizes RUNX2 activity as the central signal. In chondrocytes that are transitioning to hypertrophy, the cisCXp promoter initiates the production of $\operatorname{sh} R u n x 2$ that downregulates RUNX2, which in turn decreases the transcriptional activity of the cisCXp promoter. The negative feedback regulation of chondrocyte maturation also occurs naturally, often limited by the range of paracrine signaling (e.g., PTHrP/IHH feedback loop $)^{55-57}$. Free from the dependence on paracrine signaling, cisCXpshRunx2 gene circuits allow chondrocytes to resist maturation based their internal tendency to undergo hypertrophy, measured by RUNX2 activity. As a gatekeeper, RUNX2 mediates the signaling of many molecular and biophysical cues in chondrocytes ${ }^{4,58,59}$. Therefore, by inputting the intracellular RUNX2 activity as the signal for the negative-feedback regulation, cells expressing cisCXp-shRunx2 can dynamically adjust the level of silencing required to maintain their steady states (levels of RUNX2 suppression) without needing to address the specific pathways underlying hypertrophy stimuli, such as inflammatory factors like IL-1 $\beta$. Finally, design of cisCXp promoter allows cisCXp-shRunx2 gene circuits to provide a tunable level of RUNX2 suppression. We observed that increasing the number of $c$ is-enhancers in the gene circuit progressively enhanced RUNX2 silencing 
from $18 \%$ to nearly $80 \%$. Increasing the number of cis-enhancers also led to faster equilibrium of the activity of the gene circuits.

\section{CONCLUSION}

Overall, our circuit design is modular with a straightforward construction, which allows for simple testing of various combinations of circuit components and outputs. This flexible design enables circuits to be optimized for a variety of environmental conditions. Our results demonstrate that this approach could be a viable alternative to strategies that use broad spectrum spatial/temporal regulation of exogenous cues or constitutive or transient silencing of intracellular messengers. However, the behavior of cisCXp-shRunx2 or similar gene circuits need to be further investigated both in vitro and in vivo, with future focus on characteristics such as the promoter tunability and off-target effects.

\section{ACKNOWLEDGEMENTS}

We thank Ciara Davis, John Braford, Meghan Burns, Hannah Floyd, Samad Emory, and Sunny Karnan for their assistance in analysis of the data and Colleen Flanagan for her editorial assistance. Supported by the ANRF Award No. 310 047420, Orthopaedic Research and Education Foundation Award No. 651100, and NIH-NIAMS Award No. 1R21AR07401101. 


\section{REFERENCE}

1. Chen, H. et al. Runx2 regulates endochondral ossification through control of chondrocyte proliferation and differentiation. J. Bone Miner. Res. 29, 2653-65 (2014).

2. Nakashima, K. \& de Crombrugghe, B. Transcriptional mechanisms in osteoblast differentiation and bone formation. Trends Genet. 19, 458-66 (2003).

3. Hellingman, C. A. et al. Smad Signaling Determines Chondrogenic Differentiation of Bone-MarrowDerived Mesenchymal Stem Cells: Inhibition of Smad1/5/8P Prevents Terminal Differentiation and Calcification. TISSUE Eng. Part A 17, (2011).

4. Dong, Y.-F., Soung, D. Y., Schwarz, E. M., O’Keefe, R. J. \& Drissi, H. Wnt induction of chondrocyte hypertrophy through the Runx2 transcription factor. J. Cell. Physiol. 208, 77-86 (2006).

5. Stanton, L.-A., Sabari, S., Sampaio, A. V, Underhill, T. M. \& Beier, F. p38 MAP kinase signalling is required for hypertrophic chondrocyte differentiation. Biochem. J. 378, 53-62 (2004).

6. Shu, B. et al. BMP2, but not BMP4, is crucial for chondrocyte proliferation and maturation during endochondral bone development. J. Cell Sci. 124, 3428-40 (2011).

7. Keller, B. et al. Interaction of TGF $\beta$ and BMP Signaling Pathways during Chondrogenesis. PLoS One 6, e16421 (2011).

8. Guo, J. et al. PTH/PTHrP receptor delays chondrocyte hypertrophy via both Runx2-dependent and independent pathways. Dev. Biol. 292, 116-28 (2006).

9. Mak, K. K., Kronenberg, H. M., Chuang, P.-T., Mackem, S. \& Yang, Y. Indian hedgehog signals independently of PTHrP to promote chondrocyte hypertrophy. Development 135, 1947-56 (2008).

10. WANG, J., ZHOU, J. \& BONDY, C. A. Igf1 promotes longitudinal bone growth by insulin-like actions augmenting chondrocyte hypertrophy. FASEB J. 13, 1985-1990 (1999).

11. Zhu, M., Feng, Q., Sun, Y., Li, G. \& Bian, L. Effect of cartilaginous matrix components on the chondrogenesis and hypertrophy of mesenchymal stem cells in hyaluronic acid hydrogels. J. Biomed. Mater. Res. Part B Appl. Biomater. 105, 2292-2300 (2017).

12. Pelttari, K. et al. Premature induction of hypertrophy during in vitro chondrogenesis of human mesenchymal stem cells correlates with calcification and vascular invasion after ectopic transplantation in SCID mice. Arthritis Rheum. 54, 3254-3266 (2006).

13. Mueller, M. B. \& Tuan, R. S. Functional characterization of hypertrophy in chondrogenesis of human mesenchymal stem cells. Arthritis Rheum. 58, 1377-88 (2008).

14. Mueller, M. B. et al. Hypertrophy in mesenchymal stem cell chondrogenesis: effect of TGF-beta isoforms and chondrogenic conditioning. Cells. Tissues. Organs 192, 158-66 (2010).

15. Aisenbrey, E. A. \& Bryant, S. J. The role of chondroitin sulfate in regulating hypertrophy during MSC chondrogenesis in a cartilage mimetic hydrogel under dynamic loading. Biomaterials 190-191, 51-62 (2019).

16. Deng, Y. et al. Engineering hyaline cartilage from mesenchymal stem cells with low hypertrophy potential via modulation of culture conditions and Wnt/ $\beta$-catenin pathway. Biomaterials 192, 569-578 (2019).

17. Foyt, D. A. et al. Hypoxia impacts human MSC response to substrate stiffness during chondrogenic differentiation. Acta Biomater. 89, 73-83 (2019).

18. Brunger, J. M., Zutshi, A., Willard, V. P., Gersbach, C. A. \& Guilak, F. CRISPR/Cas9 Editing of Murine Induced Pluripotent Stem Cells for Engineering Inflammation-Resistant Tissues. Arthritis Rheumatol. 69, 1111-1121 (2017).

19. Brunger, J. M., Zutshi, A., Willard, V. P., Gersbach, C. A. \& Guilak, F. Genome Engineering of Stem Cells for Autonomously Regulated, Closed-Loop Delivery of Biologic Drugs. Stem Cell Reports 8 , 1202-1213 (2017).

20. Meerbrey, K. L. et al. The pINDUCER lentiviral toolkit for inducible RNA interference in vitro and in vivo. Proc. Natl. Acad. Sci. U. S. A. 108, 3665-70 (2011).

21. Zheng, Q. et al. Localization of the cis-enhancer element for mouse type X collagen expression in hypertrophic chondrocytes in vivo. J. Bone Miner. Res. 24, 1022-32 (2009). 
22. Atsumi, T., Miwa, Y., Kimata, K. \& Ikawa, Y. A chondrogenic cell line derived from a differentiating culture of AT805 teratocarcinoma cells. Cell Differ. Dev. 30, 109-16 (1990).

23. Shukunami, C. et al. Chondrogenic differentiation of clonal mouse embryonic cell line ATDC5 in vitro: differentiation-dependent gene expression of parathyroid hormone (PTH)/PTH-related peptide receptor. J. Cell Biol. 133, 457-68 (1996).

24. Shukunami, C. et al. Cellular hypertrophy and calcification of embryonal carcinoma-derived chondrogenic cell line ATDC5 in vitro. J. Bone Miner. Res. 12, 1174-88 (1997).

25. Wu, B. et al. Phosphate regulates chondrogenesis in a biphasic and maturation-dependent manner. Differentiation 95, 54-62 (2017).

26. Mengshol, J. A. IL-1 induces collagenase-3 (MMP-13) promoter activity in stably transfected chondrocytic cells: requirement for Runx-2 and activation by p38 MAPK and JNK pathways. Nucleic Acids Res. 29, 4361-4372 (2001).

27. Takahashi, A. et al. DNA methylation of the RUNX2 P1 promoter mediates MMP13 transcription in chondrocytes. Sci. Rep. 7, (2017).

28. Olson, A., Sheth, N., Lee, J. S., Hannon, G. \& Sachidanandam, R. RNAi Codex: a portal/database for short-hairpin RNA (shRNA) gene-silencing constructs. Nucleic Acids Res. 34, D153-7 (2006).

29. Carrion, B. et al. The Synergistic Effects of Matrix Stiffness and Composition on the Response of Chondroprogenitor Cells in a 3D Precondensation Microenvironment. Adv. Healthc. Mater. (2016). doi:10.1002/adhm.201501017

30. Kim, Y. J., Sah, R. L., Doong, J. Y. \& Grodzinsky, A. J. Fluorometric assay of DNA in cartilage explants using Hoechst 33258. Anal. Biochem. 174, 168-76 (1988).

31. Rueden, C. T. et al. ImageJ2: ImageJ for the next generation of scientific image data. BMC Bioinformatics 18, (2017).

32. Schindelin, J. et al. Fiji: An open-source platform for biological-image analysis. Nature Methods 9, 676682 (2012).

33. Murtaugh, L. C., Chyung, J. H. \& Lassar, A. B. Sonic hedgehog promotes somitic chondrogenesis by altering the cellular response to BMP signaling. Genes Dev. 13, 225-37 (1999).

34. Murtaugh, L. C., Zeng, L., Chyung, J. H. \& Lassar, A. B. The chick transcriptional repressor Nkx3.2 acts downstream of Shh to promote BMP-dependent axial chondrogenesis. Dev. Cell 1, 411-22 (2001).

35. Marcelle, C., Ahlgren, S. \& Bronner-Fraser, M. In Vivo Regulation of Somite Differentiation and Proliferation by Sonic Hedgehog. Dev. Biol. 214, 277-287 (1999).

36. Inada, M. et al. Maturational disturbance of chondrocytes inCbfa1-deficient mice. Dev. Dyn. 214, 279290 (1999).

37. Kim, I. S., Otto, F., Zabel, B. \& Mundlos, S. Regulation of chondrocyte differentiation by Cbfa1. Mech. Dev. 80, 159-70 (1999).

38. Stricker, S., Fundele, R., Vortkamp, A. \& Mundlos, S. Role of Runx Genes in Chondrocyte Differentiation. Dev. Biol. 245, 95-108 (2002).

39. Dexheimer, V., Frank, S. \& Richter, W. Proliferation as a requirement for in vitro chondrogenesis of human mesenchymal stem cells. Stem Cells Dev. 21, 2160-9 (2012).

40. Akiyama, H. et al. Positive and negative regulation of chondrogenesis by splice variants of PEBP2?A/CBF?1 in clonal mouse EC cells, ATDC5. J. Cell. Physiol. 181, 169-178 (1999).

41. Correa, D. et al. Zfp521 is a target gene and key effector of parathyroid hormone-related peptide signaling in growth plate chondrocytes. Dev. Cell 19, 533-46 (2010).

42. Komori, T. et al. Targeted disruption of Cbfa1 results in a complete lack of bone formation owing to maturational arrest of osteoblasts. Cell 89, 755-64 (1997).

43. Otto, F. et al. Cbfa1, a candidate gene for cleidocranial dysplasia syndrome, is essential for osteoblast differentiation and bone development. Cell 89, 765-771 (1997).

44. Komori, T. Runx2, A multifunctional transcription factor in skeletal development. J. Cell. Biochem. 87, 1-8 (2002).

45. Lengner, C. J. et al. Nkx3.2-mediated repression of Runx2 promotes chondrogenic differentiation. J. Biol. Chem. 280, 15872-9 (2005).

46. Tchetina, E., Mwale, F. \& Poole, A. R. Distinct phases of coordinated early and late gene expression in 
growth plate chondrocytes in relationship to cell proliferation, matrix assembly, remodeling, and cell differentiation. J. Bone Miner. Res. 18, 844-51 (2003).

47. Fosang, A. J., Last, K., Knäuper, V., Murphy, G. \& Neame, P. J. Degradation of cartilage aggrecan by collagenase-3 (MMP-13). FEBS Lett. 380, 17-20 (1996).

48. Mitchell, P. G. et al. Cloning, expression, and type II collagenolytic activity of matrix metalloproteinase13 from human osteoarthritic cartilage. J. Clin. Invest. 97, 761-768 (1996).

49. Liao, L. et al. Deletion of Runx2 in Articular Chondrocytes Decelerates the Progression of DMMInduced Osteoarthritis in Adult Mice. Sci. Rep. 7, 2371 (2017).

50. Kimura, A. et al. Runx 1 and Runx2 cooperate during sternal morphogenesis. Development 137, 11591167 (2010).

51. Lee, K. S. et al. Runx 2 is a common target of transforming growth factor beta1 and bone morphogenetic protein 2, and cooperation between Runx2 and Smad5 induces osteoblast-specific gene expression in the pluripotent mesenchymal precursor cell line C2C12. Mol. Cell. Biol. 20, 8783-92 (2000).

52. Arnosti, D. N. \& Kulkarni, M. M. Transcriptional enhancers: Intelligent enhanceosomes or flexible billboards? J. Cell. Biochem. 94, 890-898 (2005).

53. Harafuji, N., Keys, D. N. \& Levine, M. Genome-wide identification of tissue-specific enhancers in the Ciona tadpole. Proc. Natl. Acad. Sci. U. S. A. 99, 6802-5 (2002).

54. Li, F. et al. Runx2 Contributes to Murine Col10a1 Gene Regulation Through Direct Interaction With Its Cis-Enhancer. doi:10.1002/jbmr.504

55. Kronenberg, H. M. Developmental regulation of the growth plate. Nature 423, 332-6 (2003).

56. Schipani, E. et al. Targeted expression of constitutively active receptors for parathyroid hormone and parathyroid hormone-related peptide delays endochondral bone formation and rescues mice that lack parathyroid hormone-related peptide. Proc. Natl. Acad. Sci. 94, (1997).

57. Weir, E. C. et al. Targeted overexpression of parathyroid hormone-related peptide in chondrocytes causes chondrodysplasia and delayed endochondral bone formation. Proc. Natl. Acad. Sci. 93, (1996).

58. Smad-Runx Interactions During Chondrocyte Maturation : JBJS.

59. Yoshida, C. A. et al. Runx 2 and Runx 3 are essential for chondrocyte maturation, and Runx 2 regulates limb growth through induction of Indian hedgehog. Genes Dev. 18, 952-63 (2004). 
bioRxiv preprint doi: https://doi.org/10.1101/2021.04.20.440669; this version posted April 20, 2021. The copyright holder for this preprint (which was not certified by peer review) is the author/funder. All rights reserved. No reuse allowed without permission.

\section{SUPPLMENTAL MATERIALS}

Table S1. Primers for vector cloning

\begin{tabular}{|ll|}
\hline \multicolumn{1}{|c|}{ Primer } & \multicolumn{1}{c|}{ Sequence } \\
\hline shRunx2 PCR amplification $\mathrm{F}$ & 5'-CAGAAGGCTCGAGAAGGTATATGCTGTTGACAGTGAGCG \\
shRunx2 PCR amplification R & 5'-CTAAAGTAGCCCCTTGAATTCCGAGGCAGTAGGCA \\
miR30-shRunx2-miR30 PCR amplification F & 5'-GATCCAGCCTACCGGTAAGCCTTGTTAAGTGCTCGC \\
miR30-shRunx2-miR30 PCR amplification R & 5'-CTAAAGTAGCCCCTTGAATTCCGAGGCAGTAGGCA \\
1 cisCXp amplification for 1cisCXp-shRunx2 F & 5'-AAAAATTCAAAATTTATCGATCACGAGACTAGCCTCCTGTTTCACG \\
1 cisCXp amplification for 1cisCXp-shRunx2 R & 5'-CGGGCCCGCGGTACCGTCGACTGCAGAATT \\
1 cisCXp amplification for 2/3cisCXp-shRunx2 F & 5'-CAATCTGTTAGATCCGCCTCCTGTTCACG \\
1 cisCXp amplification for 2/3cisCXp-shRunx2 R & 5'-CGGGCCCGCGGTACCGTCGACTGCAGAATT \\
cis-enhancer amplification for 2/3cisCXp-shRunx2 first F & 5'-AAAAATTCAAAATTTATCGATCACGAGACTAGCCTCCTGTTTCACG \\
cis-enhancer amplification for 2/3cisCXp-shRunx2 first R & 5'-AACAGGAGGCGGATCTAACAGATTGTAGAATCAGAGTA \\
cis-enhancer amplification for 3cisCXp-shRunx2 middle F & 5'-CAATCTGTTAGATCCGCCTCCTGTTCACG \\
cis-enhancer amplification for 3cisCXp-shRunx2 middle R 5'-AACAGGAGGCGGATCTAACAGATTGTAGAATCAGAGTA
\end{tabular}

Table S2. qPCR primers

\begin{tabular}{|lllll|}
\hline \multicolumn{1}{|c}{ Gene } & & Primer Se quences $\left(\mathbf{5}^{\prime} \rightarrow \mathbf{3}^{\prime}\right)$ & \multicolumn{1}{c|}{ Primer Se quences $\left(\mathbf{5}^{\prime} \rightarrow \mathbf{3}^{\prime}\right)$} \\
\hline \hline Acan & Forward & CGCCACTTTCATGACCGAGA & Reverse & CAAATTGCAGAGAGTGTCCGT \\
Adamts 4 & Forward & ATGGCCTCAATCCATCCCAG & Reverse & AAGCAGGGTTGGAATCTTTGC \\
Adamts5 & Forward & GGAGCGAGGCCATTTACAAC & Reverse & CGTAGACAAGGTAGCCCACTTT \\
Col2al & Forward & ACGAGGCAGACAGTACCTTG & Reverse & AGTAGTCTCCGCTCTTCCACT \\
Colloal & Forward & CCAAACGCCCACAGGCATAA & Reverse & TGCCTTGTTCTCCTCTTACTGG \\
Hprt & Forward & CTGGTGAAAAGGACCTCTCGAA & Reverse & CTGAAGTACTCATTATAGTCAAGGGCAT \\
Ppia & Forward & CGCGTCTCCTTCGAGCTGTTTG & Reverse & TGTAAAGTCACCACCCTGGCACAT \\
Mmp 13 & Forward & GGAGCCCTGATGTTTCCCAT & Reverse & GTCTTCATCGCCTGGACCATA \\
\hline
\end{tabular}



Figure S1. Fold change (relative to day 0 ) in DNA content at day 7 (different groups at day 7 were compared against day 0) 